\title{
Comparative analysis of magnetic fields, low temperatures and their combined action on growth of some conditionally pathogenic and normal humane microflora
}

\author{
Olena V. Derev'yanko', Oleksandr I. Raichenko', Vladimir S. Mosienko², \\ Vladimir O. Shlyakhovenko ${ }^{2}$, Yuri V. Yanish ${ }^{2}$, Olena V. Karnaushenko ${ }^{2}$ \\ ${ }^{1}$ Frantsevych Institute for Problems of Materials Science of NASU, Kyiv, Ukraine; *Corresponding Author: 1ele@ ukr-inter.net \\ ${ }^{2}$ Kavetsky Institute of Experimental Patology, Oncology and Radiobiology of NASU, Kyiv, Ukraine
}

Received 30 July 2013; revised 25 September 2013; accepted 1 October 2013

Copyright (C) 2013 Olena V. Derev'yanko et al. This is an open access article distributed under the Creative Commons Attribution License, which permits unrestricted use, distribution, and reproduction in any medium, provided the original work is properly cited.

\section{ABSTRACT}

Growth dynamic of bacterial population after influence of magnetic field and cryoaction has been studied. In vitro experiments were performed on Staphylococcus aureus (202), Staphylococcus aureus (wild) Micrococcus lysodeicticus and Pseudomonas aeruginosa. Cryotreatment: it has been demonstrated that both Staphylococcus aureus (202) and Staphylococcus aureus (wild) Micrococcus lysodeicticus and Pseudomonas aeruginosa respond to the impact of cryoaction by pronounced growth retardation followed by rapid increase in biomass increment of cryo-resistant clones selected during the experiment. Magnetic influence: it has been shown that neither alternating magnetic field (MF) (induction $30 \mathrm{mT}$ ) nor constant MF (induction 52 $\mathrm{mT}$ ) applied separately from deep freezing have not induced any changes in further growth of both Staphylococcus aureus (202) and Staphylococcus aureus (wild) cultures. Combined action: Deep freezing (up to $-45^{\circ} \mathrm{C}$ ) achieved within 70 sec compared with the action of constant MF significantly stimulated $(P<0.05)$ growth of Staphylococcus aureus (202) and Staphylococcus aureus (202). Combined influence of cryoaction and alternating MF had no substantial effect on growth.

Keywords: Biological Objects; Magnetic Field; Cryotreatment; Bacteria

\section{INTRODUCTION}

Development of anthropogenic civilization led to new problems. The influence on the living organisms of physical and chemical factors of external environment, products of chemical and radiation contamination, vibrations of acoustic and subacoustic range, in particular, electromagnetic fields, and others take important place.

A modern man is under the mentioned influences not only by passive appearance, using a consumer electronics or transport. The physical factors affected a patient directly: during radial therapy and chemotherapy, in oncologic medical practice, in particular, by using criosurgery methods which acquire all of greater value in clinic and cosmetology.

It is impossible to forget in this sense, that the human's body practically everywhere has the normal microflora (on the whole $10^{14}$ bacteria and other microorganisms), here and there changed a pathological process or direct infection during a sharp disease. Microorganisms accompany us during all of life (and some time after). They are involved in the production links of microbiological and food industry. Therefore researches directed on suppression of harmful and stimulation of useful microbes are actual problems.

\section{MATERIALS AND METHODS}

Combined influence of low temperatures and magnetic field was performed with using of bacterial culture suspensions. Representatives of the microflora, occurring in postoperative wounds with septic complicated healing, 
and normal microflora from human large intestine were used as test objects $[1,2]$. We carried out our experiments with two opportunistic cocci (Staphylococcus aureus, strain 202 and Staphylococcus aureus, strain wild) as well as nonpathogenic spore-forming microorganism, Bacillus cereus, and blue pus bacillus Pseudomonas aeruginosa. Normal microflora was represented by Lactobacillus delbrueckii. Bacteria were suspended in distilled water in compliance with all requirements of sterility.

The value of magnetic field used in experiments was $50 \mathrm{mT}$ for constant $\mathrm{MF}$ and $30 \mathrm{mT}$ for alternating MF. Temperature range from $-20^{\circ} \mathrm{C}$ to $-45^{\circ} \mathrm{C}$ was used in the experiments in vitro. Magnetic field was applied at both freezing and thawing stages. Exposition of magnetic field applied was in the range of $120-900 \mathrm{sec}$.

To affect microorganisms by MF, the samples of bacterial suspension (volume $0.5-1 \mathrm{ml}$ ) in Eppendorf tubes of total volume of $1.5 \mathrm{ml}$ were placed between the poles of electromagnet. In case of control and in the experiments when cryoaction was studied only, electromagnet was switched off. Sterile applicator, which brings liquid nitrogen, was immersed in the sample to a depth of 10 $\mathrm{mm}$ measured from the top of the meniscus of bacterial suspension.

Cryoaction on bacterial suspensions consisted of their cooling to $-45^{\circ} \mathrm{C}$ and storing for $70 \mathrm{sec}$. Thawing procedure took place in free regime at room temperature and lasted approximately 10 min each time.

In case of separate action of MF on bacteria, two regimes of magnetization (120 sec or $900 \mathrm{sec})$ were applied. Since there was no difference in results, exposition of both alternating and constant MF in further experiments was chosen to be $120 \mathrm{sec}$. In case of combined influence of MF and cryoaction, MF was applied only during freezing of the samples plus another $50 \mathrm{sec}$ after thawing with gradual elevation of temperature inside the tube.

After thawing, samples exposed to cryoaction or combined influence of MF and deep freezing, were divided into aliquots and then suspensions of both stains of Staphylococcus aureus and Bacillus cereus were sown in standard Petri dishes onto solid nutrient medium (ribopeptone agar, RPA). With regard to Bacillus cereus, non-frozen samples (control and samples exposed to MF only) were subjected to the same procedure. As a rule, results of experiments were calculated on a day after sowing of material, and if it is necessary, to 7 consecutive days.

Staphylococci and Bacillus were cultivated at $37^{\circ} \mathrm{C}$, Lactobacillus was cultivated at $39^{\circ} \mathrm{C}$ (cultivation was carried out on liquid manufactured nutrient medium MRS). The latter culture was diluted with distilled water at a ratio of 1:25 before spectrophotometrical measurement.

Growth rate of bacteria cultivated on RPA (by 4 standard grades:,,,++++++++++$)$ or absence of growth (-) was determined visually. In addition, we estimated character of growth, the average number of colonies per 1 $\mathrm{cm}^{2}$ and average diameter of colonies $(\mathrm{mm})$.

Growth of Lactobacillus was tested by changes in optical density of culture in liquid medium. Spectrophotometrical analysis at 440, 490 and $540 \mathrm{~nm}$ was used because difference between values of optical absorption of control and experimental samples measured at these wave lengths appeared to be the most informative.

\section{DISCUSSION}

It has been demonstrated that constant MF has no effect on growth of both strains of Staphylococcus aureus (202 and wild). Similarly, all above-mentioned microorganisms were not affected by alternating MF (Tables 1 and 2; Figure 1).

Cryoaction in all studied cases caused growth retardation of both Staphylococci and Bacillus cereus during the period of 1 - 2 days with the following growth restoration of selected cryostable subpopulation. Since cultural peculiarities of microorganisms survived at rapid freezing have to be interrogated, we preliminarily concluded that such an unexpected selection should be taken into consideration during cryomagnetic therapy of patients.

Intense growth stimulation of Staphylococcus aureus (202) induced by combined action of constant MF and freezing in comparison with intact control has been shown. On the other hand, combined influence of alternating MF and cryoaction had no effect on Staphylococcus aureus (wild) compared with freezing action alone (Tables 1 and 2; Figure 1).

Table 1. Influence of constant magnetic field (MF), deep freezing and their combination on growth of Staphylococcus aureus (202).

\begin{tabular}{cccc}
\hline Type of influence & Intensity of growth over 1 day & A number of colonies per $1 \mathrm{~cm}^{2}$ of RPA surface & Colony diameter, mm \\
\hline Intact control & ++ & $127 \pm 20$ & $0.21 \pm 0.02$ \\
Post-MF & ++ & $117 \pm 20$ & $0.20 \pm 0.01$ \\
Cryo & + & $28 \pm 6$ & $0.69 \pm 0.07$
\end{tabular}


Table 2. Influence of alternating magnetic field (MF), deep freezing (Cryo) and their combined action (AltMF + Cryo) on growth of Staphylococcus aureus (wild).

\begin{tabular}{cccccc}
\hline Type of influence & \multicolumn{2}{c}{ Intensity of growth over } & $\begin{array}{c}\text { A number of colonies per } 1 \mathrm{~cm}^{2} \text { of RPA surface } \\
\text { on the 7-th day }\end{array}$ & Colony diameter on the 7-th day, mm \\
\hline & 1 Day & 4 Days & 7 Days & & $0.96 \pm 0.05$ \\
Intact control & +++ & +++ & ++++ & $74 \pm 7$ & $0.66 \pm 0.07$ \\
AltMF & +++ & +++ & ++++ & $77 \pm 9$ & $0.92 \pm 0.09$ \\
Cryo & - & ++ & +++ & $27 \pm 3$ & $0.84 \pm 0.04$ \\
AltMF + Cryo & - & ++ & +++ & $39 \pm 3$ & \\
\hline
\end{tabular}

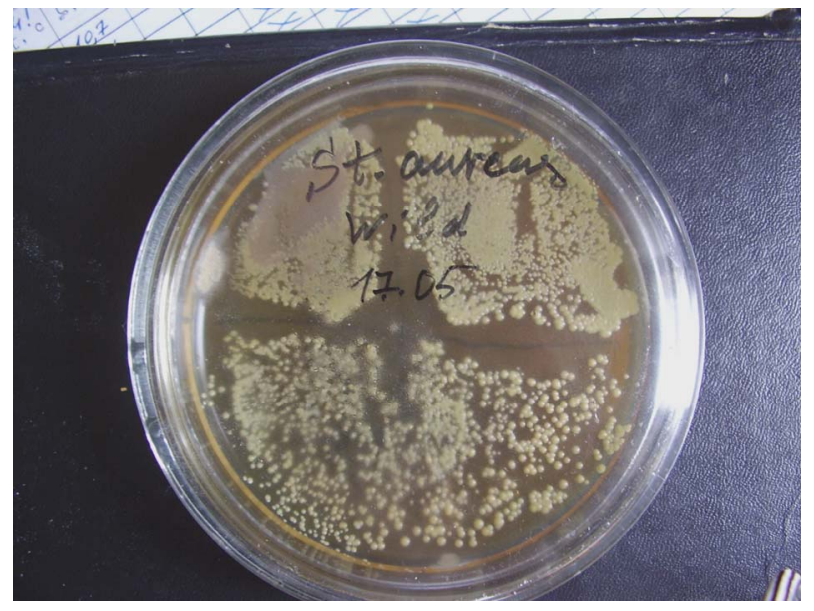

Figure 1. Influence of alternating magnetic field, deep freezing and their combined action on growth of Staphylococcus aureus (wild) culture: upper right quadrant - control; lower right quadrant-alternating MF; left lower quadrant-cryoaction; left upper quadrant - cryomagnetic action.

It has been shown that neither alternating MF nor constant MF have not induced any changes in further growth of both, Micrococcus lysodeicticus and Pseudomonas aeruginosa cultures. However deep and rapid freezing led to cryo-resistant clones appear ance. Since cultural features and degree of pathogenicity of novel sub-strains were not specifically examined, it should be taken into consideration that cryogenic therapy in clinics may attend appearance of microor microorganisms with unidentified antibiotic susceptibility.

It should be noted that, as numerous experiments testify on the cultures of blasts, complete death of all of cellular elements after one freezing cycle of does not take a place usually [3].

Authors reported [4] about research of subzero temperatures and MT combined action on some types of bacteria. In vitro experiments were performed on postoperative septic wound bacterial culture, and on humane large intestine bacteri-Lactobacterium delbrukii, and also - on Bacillus cereus. Bacillacae bacterium family (and Bacillus cereus as representative), is spore-forming bacteria which bear drying in a vacuum, extremely low and high enough temperatures (spores perish only at $120^{\circ} \mathrm{C}$ ). Appearing in favorable conditions, pathogenic microbes proceed in the viability and ill properties, becoming reason of such heavy diseases of animals and people, as anthrax, botulism, tetanus, emphysema but other. Bacillus cereus in our experiments is not pathogenic.

Our experiments proved that absorbancy of Lactobacteriu delbuckii suspension by comparison to control after Cryo does not change practically, after magnetic influence a bit grows (approximately on $8 \%$ ). After combined action diminishes substantially (approximately on 25\%) [4]. The amount of living microorganisms of Staphylococcus aureus (wild), by comparison to control, after Cryo (approximately in 3 times) decrease sharply. After magnetic influence remains practically unchanging, and after combined action (approximately twice) decrease substantially.

Besides, the most interesting result was obtained on the culture of Bacillus cereus [4].

When applied separately, cryoaction and magnetic field were on able to kill this microorganism, whereas combined action of these factors led to the complete devitalization of bacterial culture.

The regularity of freezing process and magnetic fields action on the biological systems is fundamental basis of these information study [5]. Staphylococcus aureus, Pseudomonas aeruginosa and some cultures of Micrococcus (luteus and roseus) is marked as a pathogen which accompanies the terminal stages of oncologic process and worsens his course [6].

On the whole, afore-mentioned experiments have very important character not only in the question of humane ecology, of health protection and improvement of his life quality. Development of industry conduces to appearance of waste, including such, which contain new anthropogenic components. In sense that authors succeeded to set some decline of survivability of some malignant bacteria, these experiments can be useful to the biotechnologies which disinfect these waste. Authors hope that as a result of deepening of such researches it will be succeeded to extend the list of malignant microorganisms which can 
be disinfect by described and other physical methods.

\section{CONCLUSIONS}

- It has been shown that neither alternating magnetic field (MF) (induction $52 \mathrm{mT}$ ) nor constant MF (induction $30 \mathrm{mT}$ and frequency $50 \mathrm{~Hz}$ ) applied separately from deep freezing have not induced any changes in further growth of both Staphylococcus aureus (202), Staphylococcus aureus (wild), Micrococcus lysodeicticus and Pseudomonas aeruginosa cultures.

- Deep freezing (up to $-45^{\circ} \mathrm{C}$ ) achieved within $70 \mathrm{sec}$ has suppressed intensity of growth of Staphylococcus aureus (202), Staphylococcus aureus (wild) Micrococcus lysodeicticus and Pseudomonas aeruginosa cultures. However, combined with alternating MF, this factor significantly stimulated $(\mathrm{P}<0.05)$ growth of Staphylococcus aureus (202 and wild). Combined Staphylococcus aureus influence of cryoaction and alternating MF had no substantial effect on growth Staphylococcus aureus compared with the action of deep freezing applied alone.

- The amount of alive microorganism Staphylococcus aureus (wild) after cryoaction decrease sharply (approximately in 3 times). It has been demonstrated that both (202) and (wild) Micrococcus lysodeicticus and Pseudomonas aeruginosa respond to the impact of cryoaction by pronounced growth retardation followed by rapid increase in biomass increment of cryo-resistant clones selected during the experiment. Since cultural features and degree of pathogenicity of novel sub-strains were not specifically examined, it should be taken into consideration that cryogenic therapy in clinics may attend appearance of microorganisms with unidentified characteristics.

The constant and alternating MFs affect bacteria in different ways. This fact can be explained by high resistance of procaryotic organisms towards influence of various surrounding factors. To summarize, our data are meant to stimulate further study in this area and perspectives of their practical application.

\section{REFERENCES}

[1] Buchanan, P.E. and Gibbons, K.E. (1974) Bergeu's manual of determinativ bacteriology. 8th Edition, Williams S. Wilkins, Baltimore.

[2] Smirnov, V.V., Reznik, S.R. and Sorokulova, I.B. (1980) Methodical recommendations for isolation and identification of Bacillus subtilis-Mesentericus bacteria group from human and animal organisms. Zdorov'e, Kiev, 26. (in Russian)

[3] Afanas'eva, N.I., Shevtsov, V.Ch. and Mysghychuk, A.V. (2002) Perspectives of application of cryo-surgery in oncology. The International Medical Journal, 1-2, 156-159.

[4] Raichenko, O.I., Mosienko, V.S., Shlyakhovenko, V.O., Derev'yanko, O.V., Yanish, Y.V. and Karnaushenko, O.V. (2012) Combined action of low temperature and magnetic field of different intensities on growth of some bacterial species in vitro. Health, 4, 249-252.

[5] Binhy, V.N. (2002) Magnetobiology: Underlying physical problems. Academic Press, San Diego.

[6] Clark, H.R. (1995) The cure for all diseases. New Century Press, Chulu Vista. 